\title{
SISTEMAS MULTIMEDIA BASADOS EN PROTOCOLO IP IMS APLICADOS A SERVICIOS LTE DE 4G
}

Edgar Alfonso Cipagauta Pedraza

Estudiante de la especialización en telecomunicaciones móviles Instituto Geográfico Agustín Codazzi ecipagauta@igac.gov.co

Bogotá, Colombia

\section{Roberto Ferro Escobar}

Ingeniero Electrónico

Universidad Distrital Francisco José de Caldas

rferro@udistrital.edu.co

Bogotá, Colombia

Tipo: Artículo de reflexión

Fecha de Recepción: Junio 15 de 2012

Fecha de Aceptación: Agosto 9 de 2012

\section{IP-IMS-BASED MULTIMEDIA SYSTEMS APPLIED TO SERVICES}

\begin{abstract}
This article presents a system intended to support multimedia services operating on New Generation Networks (NGN), namely IP Multimedia Subsystem (IMS). The article also explains the connection between IMS and the Long Term Evolution (LTE) technologies defined by $3 \mathrm{GPP}$ release 8 . IMS consists in a network-core integrated architecture that offers video-and-audio multimedia services over a single infrastructure; thus a whole range of services are now offered though IP and IMS. This technology is to become the bedrock upon which future multimedia services operate and grow. Although LTE appears as a promoter of IMS, some other factors also begin to favor the technology and to set convenient business models around such multimedia architecture. IMS is intended to enable service convergence by permanently combining the Internet with mobile communications in every corner of the globe.
\end{abstract}

Key words: 3GPP, EPC, IMS, LTE, NGN.

\section{RESUMEN}

Este artículo presenta de una manera sencilla, el sistema creado para soportar los servicios de multimedia dentro de red de nueva generación (NGN) llamada IP Multimedia Subsystem (IMS) a la vez que se explica su relación con la tecnología Long Term Evolution (LTE) definida por el 3GPP en su release 8. IMS se trata de una arquitectura integrada en el núcleo de red para ofrecer servicios multimediales de audio y video sobre una infraestructura. Entonces ahora, los servicios prestados pasarán a ser ofrecidos a través de IP IMS y será la piedra angular en donde estos servicios se generarán y se ofrecerán. LTE es sin duda un impulsor de IMS, pero hay otros factores que empiezan a favorecer y a plantear un modelo de negocio favorable para la arquitectura multimedia. IMS está orientado a habilitar la convergencia de servicios, combinando el crecimiento de la Internet con el de las comunicaciones móviles, desde cualquier ubicación y método de forma continua y permanente.

Palabras claves: 3GPP, EPC, IMS, LTE, NGN. 


\section{INTRODUCCION}

Con la llegada de la tecnología LTE las redes de los operadores sufren una transformación importante con respecto a sus redes existentes de 2G y 3G. Esta transformación no sólo pasa por el acceso de radio y el empleo de otra tecnología como OFDM, en lugar de WCDMA, sino que el ambiente LTE está dominado por IP. Con la integración total de IMS la conmutación de circuitos desaparece, no así la necesidad de ofrecer los servicios que dicha arquitectura ofrecía y que siguen siendo los más importantes en cuanto a ingresos de los operadores.

El objetivo principal de la tecnología LTE es la de mejorar las actuales redes móviles basadas en UMTS, HSDPA, HSUPA y HSPA+. Entre sus objetivos pueden citarse una mejora de la eficiencia del espectro, costos por byte transmitido más económicos, mejora en los servicios y una mejor integración con los estándares de telecomunicaciones ya existentes.

Las proyecciones entregadas por las más recientes investigaciones de ABI Research advierten que en el año 2013 [1], LTE tendrá más de 32 millones de terminales en uso en todo el mundo, esto suponiendo que el inicio de penetración del mercado sea en el actual año, dando como resultado dos años de un índice de crecimiento favorable para las inversiones generadas por las operadoras en este negocio que han invertido ya en esta tecnología.

A su vez se observa que IMS es una plataforma de servicios que permite a los operadores apoyar las aplicaciones multimedia IP. Estas aplicaciones potenciales incluyen Voz sobre IP, IPTV, streaming de vídeo y aplicaciones interactivas de juego solo por mencionar algunos ejemplos. Pero IMS por sí sola no proporciona todas estas aplicaciones, por el contrario, proporciona un marco de servidores de aplicaciones, bases de datos de suscriptor y puertas de acceso a los hacen posibles. Los servicios dependerán de los operadores celulares y la aplicación los desarrolladores que hacen que estas aplicaciones estén disponibles para los operadores. En la práctica, IMS proporciona una serie de ventajas como lo son el que proporciona una plataforma común, capaz de reducir el tiempo de puesta en marcha y salida al mercado de nuevos servicios multimedia, permite a los proveedores adoptar servicios creados por terceros y proporcionar a su vez otros nuevos que se integren fácilmente con los ya existentes. Por último, permite a los operadores decidir el tipo de facturación que debe aplicarse a cada sesión en función del servicio solicitado por el usuario. Es decir, si se factura en función del número de datos transmitidos, de la duración de la sesión o de cualquier otro criterio establecido por el operador.

Entonces LTE será la clave para la masificación del internet móvil, servicios como la transmisión teórica de datos a más de $100 \mathrm{M}$ y videos de alta definición, gracias a las tecnologías IMS y OFDMA.

La modulación elegida por el estándar 3GPP para la interfaz de acceso hace que las diferentes tecnologías de antenas MIMO tengan una facilidad de implementación, esto favorece según el medio de hasta cuadruplicar la eficacidad de transmisión de datos.

\section{SUBSISTEMA MULTIMEDIA BASADO EN PROTOCOLO IP}

\subsection{Origen de IMS}

"IMS fue introducido por los operadores móviles con el fin de facilitar el acceso ubicuo a los servicios multimedia en redes de acceso móviles e inalámbricas con calidad de servicio garantizada, con personalización de servicios, con posibilidades de facturación, y con otras características que Internet no ha podido proporcionar" [2].

IMS fue originalmente definido por el 3GPP como parte de su arquitectura de redes celulares de tercera generación, desde el release 5 hasta la última actualización hecha en la sección Ts 23.228 en el release 7 y 8 [3]. En una arquitectura de tres planos (transporte, control y servicios), IMS se ubica principalmente en el segundo plano, y su función es la gestión y el control de servicios multimedia IP, los cua- 
les están alojados en servidores de aplicación, ubicados en el plano de servicios de la misma arquitectura. La división en tres planos, tiene como ventaja la independencia relativa entre los servicios que puede ofrecer el operador y la tecnología de la red de acceso que utiliza el usuario posibilitando que IMS haya sido incorporado en otras estandarizaciones como 3GPP2 con ligeras modificaciones logrando proveer servicios convergentes y ubicuos.

La operación de IMS se basa en un conjunto de protocolos estandarizados por el Internet Engineering Task Force, (IETF), como Session Iniciation Protocol (SIP), Diameter y Megaco, protocolos que en principio fueron propuestos para redes IP, particularmente Internet, y no para redes telefónicas móviles, síntoma inequívoco de la convergencia entre Internet y las redes de los operadores de telecomunicaciones.

En resumen, "IMS es una arquitectura de control de servicios global, independiente del acceso y basada en estándares de conectividad IP, que habilita a los usuarios finales diversos tipos de servicios multimedia usando protocolos comunes de Internet [4]".

El proceso de estandarización de los servicios implementados sobre la infraestructura IMS lo lleva a cabo la organización Open Mobile Alliance (OMA).

\subsection{Arquitectura de IMS}

La arquitectura de IMS ha ido transformándose y haciéndose más universal, en la medida que los esfuerzos conjuntos de las organizaciones de estandarización tratan de cubrir los requerimientos que imponen los distintos actores del mundo de las telecomunicaciones partiendo de una estructura NGN como la mostrada en la figura 1 y un esquema básico de las capas en las que tiene influencia IMS en las NGN en la figura 2.

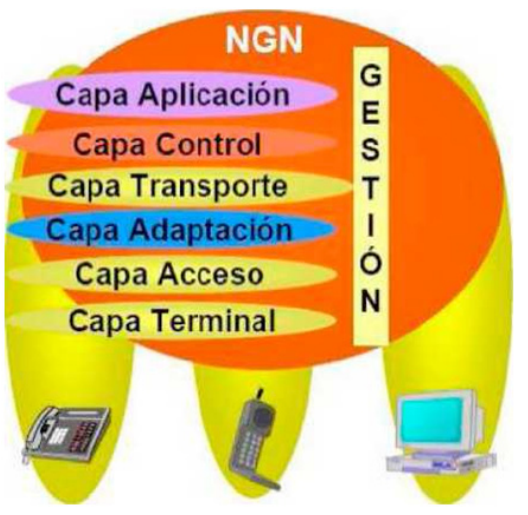

Figura 1. Enfoque de gestión por capas de NGN, donde se visualiza más fácilmente las estrategias para una fácil comprensión de las áreas de trabajo del IMS [5].

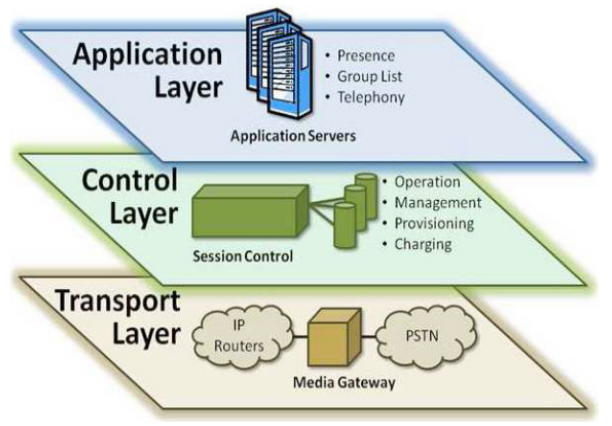

Figura 2. Esquema básico de las tres capas de influencia de la arquitectura IMS en los estándares de las NGN [6].

Capa de transporte: se ocupa de iniciar y terminar sesiones SIP, además de convertir los datos transmitidos entre formatos analógicos ó digitales y un formato basado en paquetes IP. Es la capa encargada de permitir la comunicación entre los dispositivos IMS y los dispositivos pertenecientes a la red PSTN u otras redes basadas en conmutación de circuitos.

Capa de control: los servidores o proxys SIP se denominan Call Session Control Function (CSCF) y constituyen uno de los elementos centrales de la capa de control. Son los encargados del registro SIP de los dispositivos conectados y de procesar las señales SIP enviadas por el servidor de aplicaciones. Otro de los elementos básicos en la capa de transporte es la base de datos HSS (Home Subscriber Server). Ésta se encarga de almacenar información que puede incluir direcciones IP, registros de telefonía, listas de amigos, etc. En lo que se denomina el 
perfil de servicio, único para cada usuario.

Capa de aplicación: las dos primeras capas se encargan de proporcionar una plataforma de red estandarizada que permita a los proveedores de servicios ofrecer servicios multimedia en esta capa. En la capa de aplicación, los servidores son los responsables de almacenar y ejecutar dichos servicios, así como de proporcionar su interfaz sobre las capas de control.

\subsection{Al interior del " IMS core "}

La transferencia de datos entre usuarios IMS se organiza en sesiones.

El CSCF es responsable del control de la sesión usando SIP y de las siguientes funciones:

- Autenticación del usuario.

- Enrutamiento de la llamada.

- Establecimiento de QoS en la red IP.

- Control y generación de los registros de tarificación CDRs (call detail records).

Se definen tres tipos de nodos CSCF que cumplen diferente función cada uno:

- P-CSCF (Proxy CSCF)

- I-CSCF (Interrogating CSCF)

- S-CSCF (Serving-CSCF)

\subsubsection{Proxy call state control function (P-CSCF)}

Esta entidad es el primer punto de contacto con el Core IMS; todo el tráfico de señalización SIP desde y hacia los equipos de usuario pasan por aquí. Implementa las funciones de protección de señalización, seguridad y el control de recursos del subsistema de transporte.

En el modo roaming es el nodo en la red visitada el que se encarga de enrutar la señalización de registro y sesión desde los terminales que se encuentran en situación de itinerancia hasta la red IMS nativa. Además, ejecuta las funciones comunes a los demás CSCF: el procesado y enrutado de señalización, la consulta del perfil de usuario en el HSS y la tarificación.

\subsubsection{Interrogating call state control function (I-CSCF)}

Es el primer punto de contacto dentro de la red del operador. Se encarga de contactar al HSS para obtener la dirección del S-CSCF que servirá al usuario para registrarse y reenvía requerimientos y respuestas SIP a esta entidad. Ayuda a otros nodos a determinar el siguiente salto de los mensajes SIP y a establecer un camino para la señalización. También efectúa funciones de ocultación de la topología de la red IMS ante redes externas, de forma que los elementos ajenos a IMS no puedan averiguar cómo se gestiona la señalización internamente (por ejemplo, el número, el nombre y la capacidad de los CSCF).

\subsubsection{Serving call state control function (S-CSCF)}

Realiza el control y mantenimiento de las sesiones de usuarios para acceder a servicios. A cada usuario registrado en IMS se le asigna un S-CSCF, el cual se encarga de enrutar las sesiones destinadas o iniciadas por el usuario.

También realiza el registro y autenticación del abonado IMS y la provisión de los servicios IMS (mediante el desvío de señalización a los servidores de aplicación). Asimismo aplica las políticas del operador de red y genera los registros de tarificación. Dentro de la red de un operador de red, pueden existir varios SCSFCs que pueden tener diferentes funciones.

El S-CSFC decide que servidor de aplicación es requiere para recibir la información relacionada con un requerimiento entrante de sesión SIP para asegurar el apropiado manejo del servicio. Para esto se vale de la información recibida por el home subscriber server (HSS).

\subsubsection{Home subscriber server (HSS)}

Es el equivalente del HLR en sistemas $2 \mathrm{G}$ pero extendido con dos puntos de referencia basados en el protocolo Diameter. Es la base de datos maestra de IMS que almacena los perfiles de usuario, incluyendo información de filtrado, de 
estatus y perfiles de servidores de aplicación.

El HSS almacena y gestiona el perfil del servicio IMS del abonado, almacena las claves de seguridad y genera vectores de autenticación, registra el estado de los abonados y almacena el nodo S-CSCF con el que el abonado se ha registrado.

\subsubsection{Application server (AS)}

Proporciona la plataforma de servicios en entornos IMS. Estos servidores no indican cómo son programadas las aplicaciones multimedia; solamente definen las interfaces de señalización y administración basados en SIP y Diameter. Esto permita a los desarrolladores a utilizar prácticamente cualquier paradigma de programación dentro de un servidor de aplicación SIP.

El AS es activado por el S-CSFC, el cual redirecciona algunas sesiones a él basado en los criterios dados por el HSS. El AS toma las reglas de filtrado para decidir cuál de la aplicaciones alojadas en el servidor debe ser seleccionada para manejar la sesión. Durante la ejecución de la lógica del servicio, es posible que el AS se comunique con el HSS para obtener información adicional sobre el usuario o para ser notificado de cambios en el perfil del mismo.

\subsubsection{Media resource function (MRF)}

Este puede ser dividido en varios componentes: el media resource function controller (MRFC) y el media resource function processor (MRFP) que proporcionan los recursos para el procesamiento de flujos multimedia como mezclado, anuncios, análisis y transcodificación; los border gateway control function (BGCF), media gate control function (MGCF) y media gate (MG), realizan la interconexión entre portadores RTP (real time protocol) y los portadores usados en redes tradicionales [7].

En la figura 3 se ha omitido una serie de conjuntos de entidades funcionales por sencillez, pero que están definidas por 3GPP.

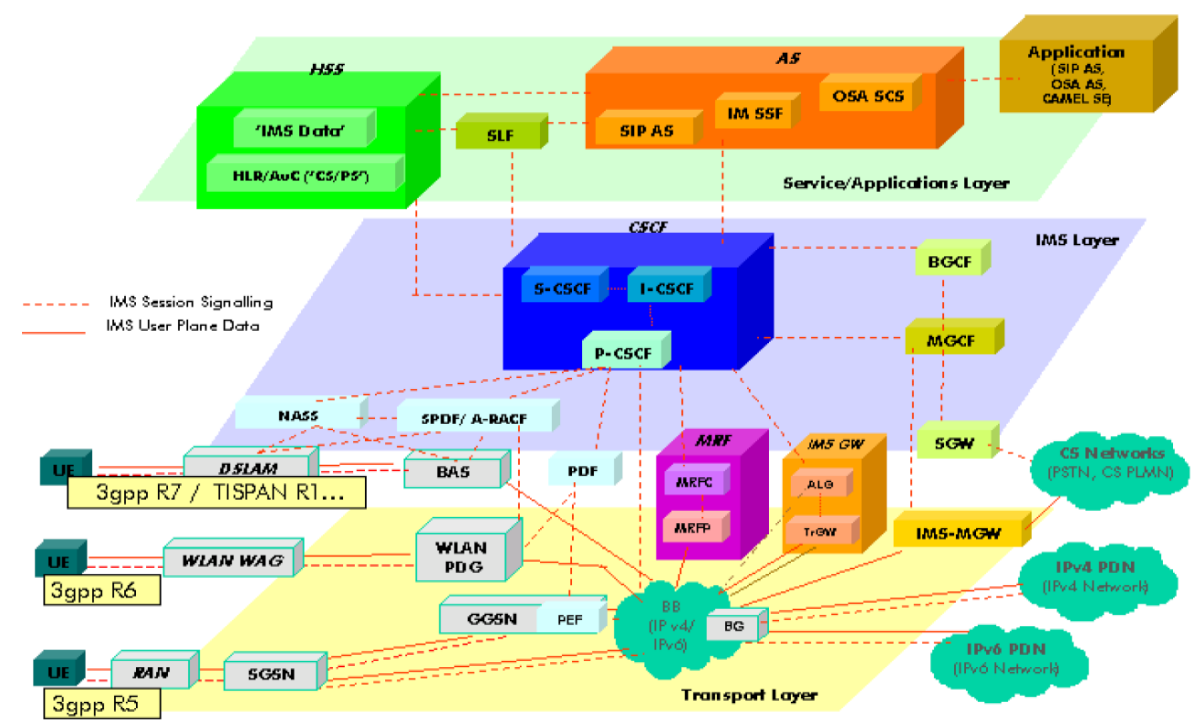

Figura 3. Detalle de nodos participantes en la arquitectura IMS según los estándares del 3GPP [8].

\section{EJEMPLO DE SERVICIO DE IPTV EN IMS}

El mercado de la televisión se está convirtiendo cada vez más importante para los operadores de telecomunicaciones con la introducción de la IPTV, siendo una oportunidad comercial para la atracción de nuevos clientes y sobresalir entre sus competidores. Para los operadores de telecomunicaciones, el desarrollo de servicios de IPTV en una plataforma unificada de servicios para reducir el OPEX y CAPEX (costos de mantenimiento de la red actuales y futuros 
respectivamente) y para introducir nuevos servicios y características del servicio son muy importantes. IMS parece haber sido adoptada por la mayoría de los operadores como la plataforma de servicio unificado para las NGN y por ende para el servicio IPTV.

\subsection{Estructurando una plataforma IPTV basada en IMS}

Inicialmente se analizan las principales características del sistema a desarrollar que son:

- Gestión de sesiones realizadas en el núcleo IMS y el servidor de aplicaciones IPTV.

- Personalización de los servicios disponibles basados en identidades de los usuarios de IMS.

- Adopción de parámetros de calidad de servicio (QoS) al dispositivo terminal utilizado para acceder a servicios de IPTV.

El entorno IMS debe soportar servicios multimedia como IPTV donde a los usuarios se muestran en una interfaz gráfica fácil de usar de acuerdo a las preferencias del usuario y las capacidades de la pantalla del terminal. Un usuario registrado puede hacer una búsqueda para un servicio específico, modificar sus preferencias, y así sucesivamente. Al hacer clic en el icono correspondiente o en el menú del servicio seleccionado es suficiente para iniciar el establecimiento de la sesión multimedia.

Durante el establecimiento de la sesión sobre la base de los mecanismos básicos del SIP, los parámetros de QoS multimedia seleccionados están adaptados al terminal del usuario actual en términos de las velocidades de bits de audio y vídeo y la resolución de vídeo. Durante el período de traspasos o handover, los parámetros de QoS se adaptan a la nueva terminal del dispositivo.

IMS service control (ISC) es la interfaz para comunicarse entre el servidor de aplicaciones IPTV (figura 4) y las funciones de control de servicio de las NGN. Las funciones de control de medios (MCF) pueden controlar las funciones de despliegue de medios (MDF) también defi- nidas en las arquitecturas NGN.

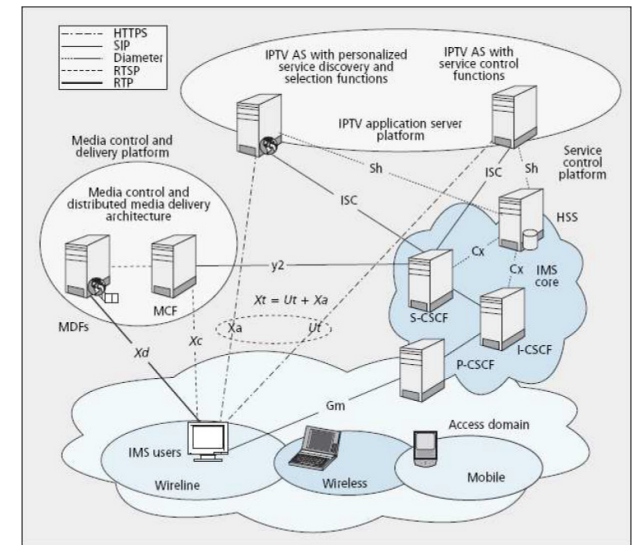

Figura 4. Arquitectura para prestación de servicios mutimediales IPTV basados en IMS [9].

En la plataforma a crear las MDF se dividen en múltiples funcionalidades específicas con tres componentes de la arquitectura se describe de la siguiente manera:

- nterconexión (I-IMDF): este elemento se encarga de la importación de medios de comunicación y el ingreso, la importación de contenido bajo demanda, los metadatos y la información de proveedor de servicios, así como la recepción de flujos en directo desde la cabecera de IPTV o de los proveedores de contenido.

- Servicio (S-IMDF): este elemento se encarga de la elaboración de contenidos (por ejemplo, la codificación, la protección del contenido, la transcodificación) y también es responsable del almacenamiento de contenidos y metadatos, así como la propagación de la información contenida en de sistemas IPTV de IMS.

- Primaria (P-IMDF): este elemento es el principal punto de contacto de los usuarios, que también proporciona las funcionalidades de streaming de todos los servicios IPTV de acuerdo con la calidad requerida para el formato, y el tipo de emisión (multi ó unibroadcasting). Este elemento también puede almacenar los más visitados activos ó contenido de usuario específico. IPTV basado en IMS también permite un control flexible de los servicios de IPTV, debido a la 
sesión de control basados en SIP. Por ejemplo, un usuario puede utilizar un terminal IMS para controlar remotamente su grabadora de IPTV. Traspasos de las sesiones activas de IPTV entre diferentes pantallas, por ejemplo, desde un ordenador portátil a un dispositivo de TV, puede ser otro escenario interesante uso de los servicios de IPTV basados en IMS.

\subsection{Implementación de prototipo IPTV basados en IMS [9]}

En la implementación del prototipo de la plataforma de IPTV basados en IMS, el Open IMS Core de Fraunhofer FOKUS [10] fue utilizado como el componente central de IMS. El cliente IPTV, que consiste en la parte de IMS de señalización y la parte de reproducción multimedia, así como el servidor de aplicaciones de IPTV se han desarrollado dentro del proyecto ScaleNet. La UE se refiere a los dispositivos finales que ejecutan Windows XP o Windows Mobile 5.0, así como software de IPTV de cliente basadas en IMS. El servidor de aplicaciones de IPTV se basa en un servidor Linux Debian. La plataforma de entrega de los medios de comunicación se basa en la arquitectura distribuida I-MDF, donde las funciones de I-MDF son basadas en SIP. Los servidores de multimedia I-MDF utilizados son po software clientes de vídeo de código abierto LAN (VLC).

Un esquema de paso a paso de la solicitud de servicios, la selección de servicios, establecimiento de la sesión, y la entrega de medios es realizado en un escenario de uso típico se describe en el esquema presentado en la figura. 5.

\subsubsection{Establecimiento de sesión}

Lo primero es iniciar un dispositivo con cliente IPTV (UE) y lograr una conexión de red para obtener los parámetros de red (como una dirección IP, la dirección de P-CSCF, etc). Entonces, la UE puede iniciar el proceso de registro con el core IMS y realizar funciones de servicio IPTV. La UE es capaz de iniciar el proceso de selección del servicio. El servidor de aplicaciones IPTV puede ser utilizado como un punto de contacto único para la selección de servicios y por lo tanto puede ser visto como un elemento clave para seleccionar e iniciar los servicios multimedia para los usuarios registrados de la IMS, así como para establecer la configuración de usuario o los parámetros de perfil de usuario por los propios usuarios finales.

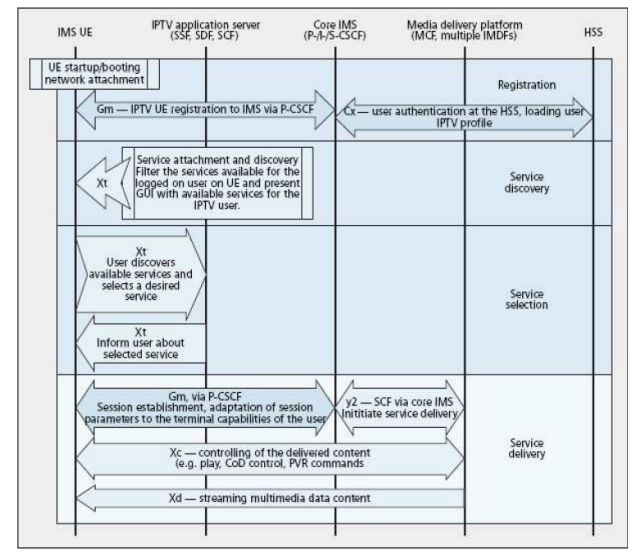

Figura 5. Procedimiento de establecimiento durante una sesión de IPTV [9].

Por lo general, una UE IMS debe saber exactamente el identificador IMS (SIP) correspondiente para establecer una sesión multimedia en el servidor IPTV mediante la generación de mensajes SIP INVITE durante el inicio del servicio. En la propuesta de ejecución, el servidor de aplicaciones de IPTV se realiza como un portal de TV en la Web. Para seleccionar el servicio deseado multimedia, el usuario puede seleccionar un elemento de un menú personalizado con la opción de informar a la UE sobre la identificación del contenido seleccionado (por ejemplo, más de una conexión http segura a través de la interfaz virtual Xt). Entonces el usuario IMS puede de forma automática o manual iniciar la sesión IPTV seleccionada utilizando la técnica mejorada de señalización SIP. La nueva sesion se establecerá desde la UE como una sesión IMS usual a través del Gm sobre el núcleo IMS. El núcleo IMS puede iniciar un proceso de reserva para los recursos de red que son requeridos para el stream de IPTV de acuerdo a las capacidades de la UE. La reserva se realiza mediante las funciones estándar de las NGN RACS y subsistemas NASS relacionada con el núcleo IMS. Con este fin, la primera sesión de 
IPTV debe ser establecido a través del núcleo IMS, no directamente con el servidor de MCF. Después del inicio de sesión con éxito, el SCF informa a la plataforma de entrega de medios (de control y distribución de contenidos) a través de núcleo IMS mediante la interfaz y2 para empezar a visualizar el contenido multimedia seleccionado. Después del establecimiento de la secuencia de datos inicial, el usuario puede controlar la interfaz Xc entre la UE y el servidor que realiza funciones de control de los medios. El protocolo RTSP se usa en esta interfaz para controlar la entrega del contenido solicitado con las características tales como formato de reproducción, pausa, adelante o atrás, o más rápido o más lento. El MDF realiza la entrega de los medios sobre la interfaz Xd basada en la prestación de flujo RTP.

\section{CONCLUSIONES}

La plataforma IMS fue diseñada para proveer conectividad IP a las redes de telefonía móvil y otorgar a los usuarios una amplia gama de servicios multimediales, siendo explotadas todas sus bondades en la venidera cuarta generación LTE gracias a su gran velocidad de transferencia.

IMS se compone de diversos servidores que se comunican entre si haciendo uso del protocolo de inicio de sesión SIP, desde el P-CSCF (al que se conecta un usuario con su terminal IMS) a los servidores de aplicación, que contienen servicios tales como: juegos multiusuario, transmisión de imágenes en tiempo real, videoconferencia, VoIP, mensajería instantánea, IPTV, etc.

Pese a sus virtudes IMS todavía presenta problemas de compativilidad en términos de configuración, seguridad y calidad de servicio entre otras, por lo que investigación continua es requerida para facilitar la transición a las redes NGN.

El proyecto Open IMS Core desarrollado por el instituto FOKUS de Alemania representa el punto de partida de nuevas investigaciones para el testeo de la arquitectura IMS en NGN de cuarta generación.

Para los operadores de telecomunicaciones móviles, la normalización de las soluciones de IPTV y el desarrollo de servicios de IPTV en una plataforma unificada de servicios para reducir el OPEX y CAPEX y para introducir nuevos servicios y características del servicio son muy importantes.

Las especificaciones para las funciones, interfaces, procedimientos y recomendaciones para el servicio de IPTV basado en IMS están aún en curso, más aún cuando la implementación de la tecnología LTE apenas se encuentra en proceso de entrada al acceso de los usuarios.

Referencias Bibliográficas

[1] ABI Research (2010, marzo), Transformación IP [Online]. Disponible: ht tp: // ww w. abiresearch.com/ research/1004433-IP+Transformation.

[2] Al-Begain, Khalid, IMS: A Development and DeploymentPerspective; John Wiley and Sons, Ltd. (2009).

[3] 3GPP (2011), IMS historia de éxito en 3GPP [Online]. Disponible: http:// www..3gpp.org/article/ims.

[4] Poikselka, Miikka; Mayer, Georg, The IMS IP multimedia concepts and services.

3ed. Wiley and Sons, Ltd. (2009).

[5] Hurtado, Javier Alexander, Aplicaciones de nueva generacion, camino a la convergencia [Online]. Disponible : ftp:// jano.unicauca.edu.co/tmp $/ \% \quad 5 B 15 \%$ 5DNGN-SIP.pdf

[6] IMS fórum (2010), 3GPP architecture with IMS interoperability [Online]. Disponible: http://www.imsforum.org/ content/public-documents.

[7] Ahson S; Ilyas M, IP multimedia subsystem (IMS) handbook. CRC Press, 
(2009).

[8] Wikipedia (2011), IP Multimedia Subsystem [Online]. Disponible:http:// en. wikipedia.org/wiki/IP_Multimedia Subsystem

[9] Eugene Mikoczy, IPTV Service over
IMS, IEEE Comunications magazine (2008, Mayo).

[10] Fraunhofer FOKUS (2010), Focus open IPTV ecosystem [Online]. Disponible: http://www.versant.com/pdf/FOKUS IPTV_Whitepaper.pdf. 